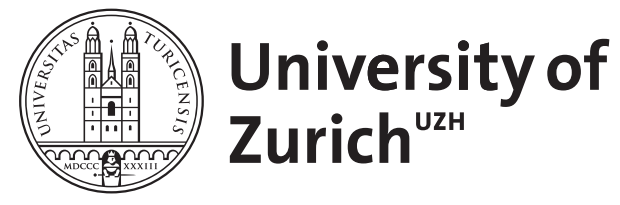

\title{
Atrial myxoma as a trigger of migraine with aura - pathophysiological considerations
}

\author{
Riederer, F ; Luft, A R ; Sándor, P S
}

DOI: https://doi.org/10.1177/0333102409360829

Posted at the Zurich Open Repository and Archive, University of Zurich ZORA URL: https://doi.org/10.5167/uzh-42998

Journal Article

Accepted Version

Originally published at:

Riederer, F; Luft, A R; Sándor, P S (2010). Atrial myxoma as a trigger of migraine with aura - pathophysiological considerations. Cephalalgia, 30(9):1149-1150.

DOI: https://doi.org/10.1177/0333102409360829 


\title{
Atrial myxoma as a trigger of migraine with aura - pathophysiological considerations
}

\author{
Franz Riederer, Andreas R Luft, Peter S Sándor ${ }^{+}$
}

Department of Neurology, University Hospital Zurich, Zurich, Switzerland

+Please address all correspondence and requests of reprints to peter.sandor@usz.ch

Letter to the editor

\section{Dear Editor,}

A 57-year-old woman had suffered from migraine with aura since childhood. Once or twice per month she experienced visual disturbances starting in the right visual field lasting about $30 \mathrm{~min}$ that were followed by strong headaches for 2-3 days accompanied by nausea. Occasionally, visual disturbances were associated with naming difficulties. Her migraine attacks became less frequent during adolescence (1-2 attacks per year). Occasionally the patient took $500 \mathrm{mg}$ paracetamol for her headaches but without much relief.

At the age of 50 years the frequency of migraine attacks with aura gradually increased. A few weeks before presenting to the emergency unit she had 1-2 attacks per week. They were associated with dizziness and bilateral lower extremity weakness and were triggered by physical exercise. Because of diplopia lasting for 3 hours during two migraine episodes, she presented at the emergency unit of a university hospital. The neurological examination revealed vertical gaze palsy. Diffusion-weighted MRI showed bilateral cerebellar infarcts and ischemic lesions in the right crus cerebri, pons and thalamus. An additional subacute infarct was noted on T2-weighted imaging in the left internal capsule. Duplex sonography of extraand intracranial arteries did not reveal any stenoses or signs of atherosclerosis. Antiplatelet therapy with ASS $300 \mathrm{mg} /$ day was initated. She was also taking predisolon $5 \mathrm{mg} / \mathrm{d}$ for recurrent pain in both calves or unclear etiology for several months. Otherwise her medical history was unremarkable. Transthoracic echocardiography revealed a $3.3 \times 2 \mathrm{~cm}$ mass in the left atrium adherent to the inter-atrial septum with partial contact to the mitral valve, suggestive of atrial myxoma. CT scan of the chest confirmed this diagnosis and additionally showed a small persistent foramen ovale (PFO). Two weeks later the myxoma was operated 
on and the small PFO was closed. The diagnosis myxoma was verified histologically. The patient was anticoagulated with phenprocoumon. Since the operation she has suffered from transient double vision once but remained free from auras and headaches (Follow-up 4 months). Interestingly, her episodes with calve pain had also disappeared.

The patient's mother had suffered from migraine without aura, the patient's daughter suffers from migraine with aura, and three nieces (daughters of one brother and 2 different sisters, respectively) suffer from migraine.

\section{Comments}

Removal of left atrial myxoma was associated with the disappearance of migraine with aura in this patient who had a very high frequency of attacks before the intervention. Cessation of migraine attacks after removal of a left atrial myxoma has been described previously (1-4). These cases show similarities with our case: (i) History of migraine with complex auras including visual, sensory, motor, or aphasic symptoms. (ii) Increase in attack frequency during adult life (1-3). (iii) Cerebral infarcts in 3 cases demonstrated on cranial MRI or CT (2$4)$.

Cerebral infarctions are known to be the most frequent neurologic complication of atrial myxoma (5). We hypothesise that cardiac emboli might have triggered cortical spreading depression (CSD) that is recognized as the pathophysiological correlate of migraine aura, in these patients. Recently it has been demonstrated in an animal model that microemboli can trigger CSD without causing brain infarctions (6). Thus, depending on the quantity and the size of these emboli, CSD alone or in association with embolic infarcts might have occurred. The fact that in our patient physical exercise constantly triggered migraine with aura further supports this hypothesis because cardiac output increases and so does the likelihood of embolus dislodgement.

Closure of PFO (7) and anticoagulation (8) have been reported to improve migraines. Either or both interventions may have contributed to migraine relief in our case. However, both measures suggest the same pathomechanism for frequent migraine in this patient, i.e., cardioembolism. Considering that the large myxoma is a more likely source of emboli than the small PFO, myxoma resection was likely to be the essential intervention here.

In the conceptual frame of a genetic predisposition to migraine and assuming that PFO and myxoma are non-heritable conditions in this patient, the long personal history of migraine with aura and the positive family history of migraine suggest that here, embolism is a trigger rather than the cause of high-frequency migraine. 
Disappearance of pain in the calves after myxoma resection suggests that this pain might have been caused by peripheral embolism.

Kern et al (3) hypothesised a causal relationship between vasoactive intestinal peptide (VIP) secreted by the myxoma and migraine with aura based on the vasoactive properties of this peptide. In our myxoma specimen VIP was negative, however, possible other endocrine effects cannot be ruled out.

We conclude that echocardiography should be considered in migraine patients with complex auras when a significant increase in attack frequency is noted.

Acknowledgement: We thank Drs. B. Helmchen and S. Lanz from Stadtspital Triemli, Zurich, Switzerland for determination of VIP.

1. Caputi L, D'Amico D, Carriero MR, Materazzo C, Parati E. Cessation of migraine with aura following removal of left atrial myxoma. Eur J Neurol 2006;13:e5-6.

2. Bowen J, John K, Pakalnis A. Left atrial myxoma presenting with migraine-like episodes. Psychosomatics 1986;27:802, 805.

3. Kern RZ, Asa S. Left atrial myxoma presenting as migraine with aura: a VIP-induced syndrome? Headache 2005;45:251-254.

4. Garnier P, Michel D, Antoine JC, et al. [Myxoma of the left atrium with neurologic manifestations: 8 cases]. Rev Neurol (Paris) 1994;150:776-784.

5. Lee VH, Connolly HM, Brown RD, Jr. Central nervous system manifestations of cardiac myxoma. Arch Neurol 2007;64:1115-1120.

6. Nozari A, Dilekoz E, Sukhotinsky I, Stein T, Eikermann-Haerter K, Liu C.

Microemboli may link spreading depression migraine aura and patent foramen ovale. Ann Neurol 2009; Published online: 14 Sep 2009. DOI 10.1002/ana.21871

7. Morandi E, Anzola GP, Angeli S, Melzi G, Onorato E. Transcatheter closure of patent foramen ovale: a new migraine treatment? J Interv Cardiol 2003;16:39-42.

8. Morales-Asin F, Iniguez C, Cornudella R, Mauri JA, Espada F, Mostacero EE.

Patients with acenocoumarol treatment and migraine. Headache 2000;40:45-47. 